\title{
The millimeter wave spectrum of methyl cyanate: a laboratory study and astronomical search in space ${ }^{\star, \star \star}$
}

\author{
L. Kolesniková1 ${ }^{\text {, J. L. Alonso }}{ }^{1}$, C. Bermúdez ${ }^{1}$, E. R. Alonso ${ }^{1}$, B. Tercero ${ }^{2}$, J. Cernicharo², and J.-C. Guillemin ${ }^{3}$ \\ 1 Grupo de Espectroscopia Molecular (GEM), Edificio Quifima, Área de Química-Física, Laboratorios de Espectroscopia y \\ Bioespectroscopia, Parque Científico UVa, Unidad Asociada CSIC, Universidad de Valladolid, 47011 Valladolid, Spain \\ e-mail: lucie.kolesnikova@uva.es \\ 2 Instituto de Ciencia de Materiales de Madrid, CSIC, C/ Sor Juana Inés de la Cruz 3, 28049 Cantoblanco, Spain \\ ${ }^{3}$ Institut des Sciences Chimiques de Rennes, École Nationale Supérieure de Chimie de Rennes, CNRS, UMR 6226, \\ 11 Allée de Beaulieu, CS 50837, 35708 Rennes Cedex 7, France
}

Received 15 January 2016 / Accepted 15 March 2016

\begin{abstract}
Aims. The recent discovery of methyl isocyanate $\left(\mathrm{CH}_{3} \mathrm{NCO}\right)$ in $\mathrm{Sgr} \mathrm{B} 2(\mathrm{~N})$ and Orion $\mathrm{KL}$ makes methyl cyanate $\left(\mathrm{CH}_{3} \mathrm{OCN}\right)$ a potential molecule in the interstellar medium. The aim of this work is to fulfill the first requirement for its unequivocal identification in space, i.e. the availability of transition frequencies with high accuracy.

Methods. The room-temperature rotational spectrum of methyl cyanate was recorded in the millimeter wave domain from 130 to $350 \mathrm{GHz}$. All rotational transitions revealed $A-E$ splitting owing to methyl internal rotation and were globally analyzed using the ERHAM program.

Results. The data set for the ground torsional state of methyl cyanate exceeds 700 transitions within $J^{\prime \prime}=10-35$ and $K_{a}^{\prime \prime}=0-13$ and newly derived spectroscopic constants reproduce the spectrum close to the experimental uncertainty. Spectral features of methyl cyanate were then searched for in Orion KL, Sgr B2(N), B1-b, and TMC-1 molecular clouds. Upper limits to the column density of methyl cyanate are provided.
\end{abstract}

Key words. astrochemistry - line: identification - techniques: spectroscopic - astronomical databases: miscellaneous molecular data

\section{Introduction}

Organic molecules containing abundant interstellar elements $\mathrm{H}, \mathrm{C}, \mathrm{N}$, and $\mathrm{O}$ are the prime target for both astrophysicists and spectroscopists to intensively search for in the interstellar medium (ISM). One of the simplest, the isocyanic acid (HNCO), was detected with high abundances in a variety of Galactic sources, as well as in the external galaxies (see Quan et al. 2010; Brünken et al. 2010, and references therein). Cyanic acid (HOCN) and fulminic acid (HCNO), two energetically less stable isomers of isocyanic acid with predicted relative energies of 24.7 and $70.7 \mathrm{kcal} \mathrm{mol}^{-1}$, respectively (see Schuurman et al. 2004), were also successfully observed in space (Brünken et al. 2010, 2009; Marcelino et al. 2010, 2009). The choice of new molecules to be searched for is usually justified by analogy with the detected molecules and plausible chemical reactions that occur in the ISM. Methyl substituted derivatives of isocyanic acid and its isomers could thus be suggested as logical candidates. Among the members of the open-chain methyl derivatives, methyl isocyanate $\left(\mathrm{CH}_{3} \mathrm{NCO}\right)$ is the lowest-energy isomer, followed by methyl cyanate $\left(\mathrm{CH}_{3} \mathrm{OCN}\right)$, acetonitrile $\mathrm{N}$-oxide $\left(\mathrm{CH}_{3} \mathrm{CNO}\right)$, and methyl fulminate $\left(\mathrm{CH}_{3} \mathrm{ONC}\right)$. Very recently, methyl isocyanate was detected on the surface of the comet

\footnotetext{
* This paper makes use of the following ALMA data: ADS/JAO.ALMA\#2011.0.00009.SV.

$\star \star$ Tables 3 and 4 are only available at the CDS via anonymous ftp to cdsarc.u-strasbg.fr (130.79.128.5) or via

http://cdsarc.u-strasbg.fr/viz-bin/qcat?]/A+A/591/A75
}

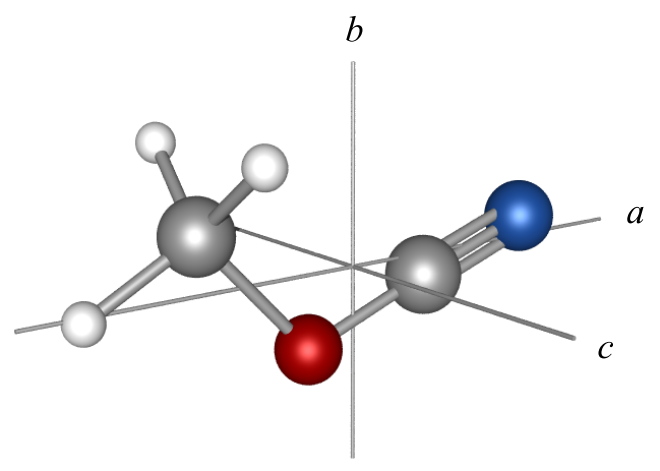

Fig. 1. Methyl cyanate depicted in the principal axis system.

67P/Churyumov-Gerasimenko (Goesmann et al. 2015) as well as in the molecular clouds Sgr B2 and Orion KL (Halfen et al. 2015; Cernicharo et al. 2016). Owing to relatively high abundances of methyl isocyanate found by Cernicharo et al. (2016), the search for methyl cyanate in space, which uses millimeter wave astronomy, looks promising. Methyl cyanate (Fig. 1) is predicted to be higher in energy with respect to methyl isocyanate by $25.1 \mathrm{kcal} \mathrm{mol}^{-1}$ (Pasinszki \& Westwood 2001), i.e. close to that of cyanic acid with respect to isocyanic acid. Moreover, sizable dipole moment components of $\left|\mu_{a}\right|=4.07$ (6) D and $\left|\mu_{b}\right|=1.24$ (40) D (Sakaizumi et al. 1990) make the possible detection of this metastable isomer relatively favourable. 

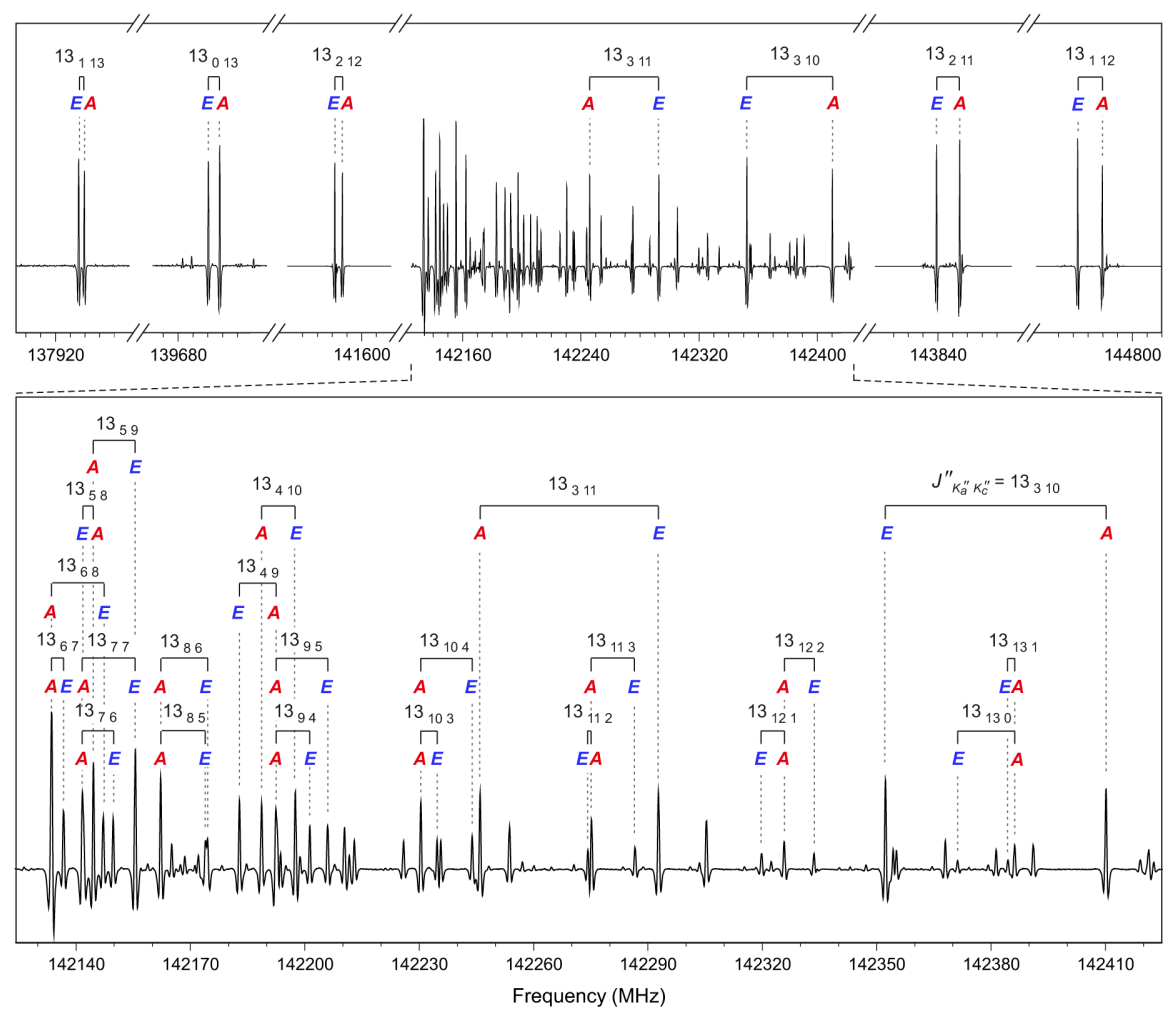

Fig. 2. Section of the methyl cyanate rotational spectrum with the assignment of the $A$ - $E$ doublets of the $a$-type $R$-branch transitions $(\Delta J=+1$, $\left.\Delta K_{a}=0, \Delta K_{c}=+1\right)$ within the cluster for $J^{\prime \prime}=13$. The quantum numbers of the lower energy levels, $J_{K_{a}^{\prime \prime} K_{c}^{\prime \prime}}^{\prime \prime}$, are indicated. Transitions of the $A$ components for $K_{a} \geq 5$ are doubly degenerate.

An attempt to observe methyl cyanate unambiguously in the ISM requires a previous good knowledge of its rotational spectrum in the laboratory. Until this work, only Stark-modulated spectra were recorded up to $50 \mathrm{GHz}$ (Sakaizumi et al. 1990, 1993, 1995). Seven $A$ and seven $E$ transitions, which resulted from the methyl group internal rotation, were reported and analyzed for the ground torsional state and a barrier to internal rotation of $399 \mathrm{~cm}^{-1}$ was derived (Sakaizumi et al. 1995). Lack of accurate laboratory millimeter wave data thus initiated new spectroscopic measurements over the frequency range from 130 to $350 \mathrm{GHz}$. A new set of spectroscopic constants could reproduce the millimeter wave spectrum within the experimental uncertainty, which could then be used to guide the search for millimeter wave signatures of methyl cyanate towards various interstellar sources. In this work, a search was conducted towards the molecular clouds Orion KL, Sgr B2(N), B1-b and TMC-1, and the results are discussed in Sect. 4.

\section{Experimental details}

Methyl cyanate was generated according to Pasinszki \& Westwood (1995) by gas-solid reaction of the evaporated O-methyl thiocarbamate $\left(\mathrm{CH}_{3} \mathrm{OC}(\mathrm{S}) \mathrm{NH}_{2}\right)$ with yellow mercury oxide ( $\mathrm{HgO}$, Sigma Aldrich) packed into a tube in half-section in a vacuum line $(0.3$ mbar pressure). A sample of O-methyl thiocarbamate was prepared according to the method given in Davies \& Maclaren (1951). To remove the water, which is a by-product, the reaction products were passed through a short tube $(15 \mathrm{~cm})$ filled in half-section with phosphorus pentoxide $\left(\mathrm{P}_{2} \mathrm{O}_{5}\right.$, Sigma Aldrich). Dried products were collected in the liquid nitrogen U-tube trap, which was then connected to the sample cell of the spectrometer. Vapour pressure of the trapped sample near $-60{ }^{\circ} \mathrm{C}$ was enough to fill the cell up to a total pressure of about $25 \mu$ bar. Undiluted methyl cyanate has been described as an extremely unstable compound (Jensen et al. 1965). Initial experiments were performed in the flow mode. However, later it was found that the measurements could be performed in the closed cell for several hours. Isocyanic acid and methyl isocyanate were found to be major impurities.

The rotational spectra were recorded at room temperature using the Valladolid millimeter wave spectrometer described in detail elsewhere (Daly et al. 2014). The measured frequency region from 130 to $350 \mathrm{GHz}$ was reached by sequential multiplication of the basic synthesizer frequency $(\leq 20 \mathrm{GHz})$ by amplifiermultiplier chains (VDI, Inc.). The synthesizer output was frequency modulated at $f=10.2 \mathrm{kHz}$ with a modulation depth of $30 \mathrm{kHz}$. The signal was detected by solid-state zero-bias detectors (VDI, Inc.) and further processed by a lock-in amplifier using $2 f$ detection. The resulting second derivative shape of the lines was fitted to the Gaussian profile function and the uncertainty of the isolated well-developed lines was estimated to be better than $50 \mathrm{kHz}$.

\section{Analysis of the spectra}

Methyl cyanate is a near-prolate asymmetric top with $C_{\mathrm{s}}$ symmetry and its molecular geometry is shown in Fig. 1. Two characteristic features can be found in the methyl cyanate rotational spectra. Firstly, the dipole moment component along the $a$-axis is much larger than the component along the $b$-axis, therefore, the rotational spectrum is dominated by strong $a$-type $R$-branch transitions. These transitions usually occur in groups with the central part situated approximately at $(B+C)\left(J^{\prime \prime}+1\right)$. Each group corresponds to specific $J^{\prime \prime}+1 \leftarrow J^{\prime \prime}$ and is formed by transitions with different $K_{a}$. Figure 2 illustrates an example of this type of group 


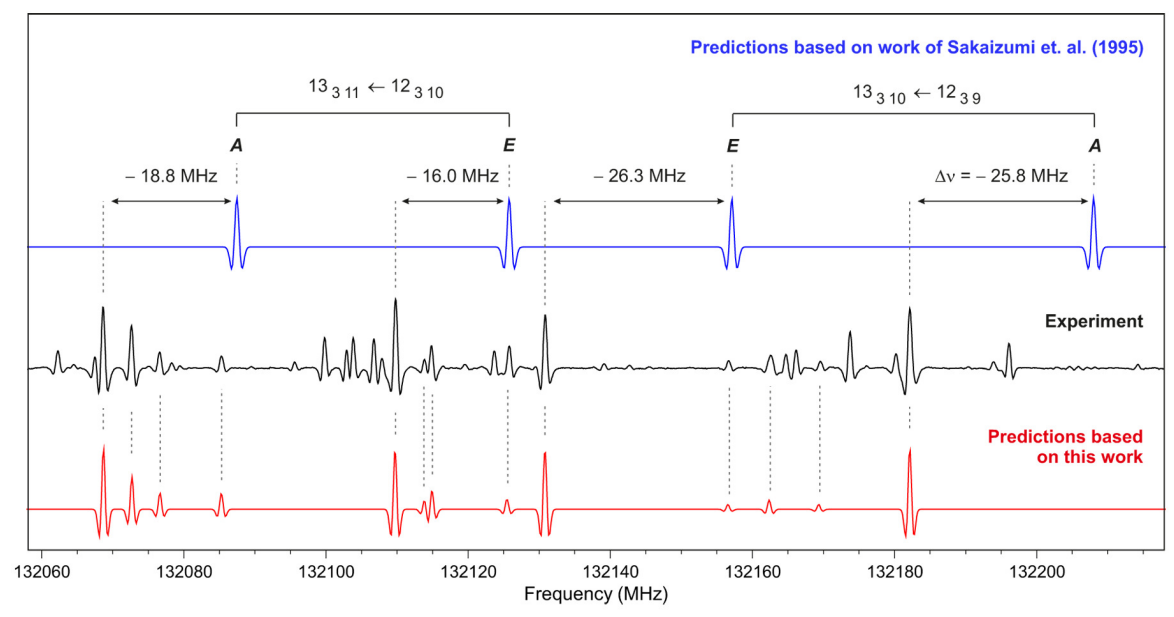

Fig. 3. Comparison of the predicted positions of the ground torsional state $a$-type $R$-branch transitions of methyl cyanate with the experimentally observed spectrum. The blue model shows predictions based on the previous work of Sakaizumi et al. (1995) and $\Delta v$ quantity provides the difference between the observed and predicted frequencies. Predictions based on the results of this work are represented by the red model. for $J^{\prime \prime}=13$. Two transitions always appear for each $K_{a}>0$ and lower $K_{a}$ pairs are more widely split than the higher $K_{a}$ ones (see Fig. 2). Secondly, methyl cyanate contains one $C_{3 v}$ internal rotor (methyl group), which can undergo a torsion motion with respect to the rest of the molecule leading to the splitting of the rotational levels into $A$ and $E$ sublevels. As a result, all transitions appear as the $A-E$ doublets with separation from units to tens of $\mathrm{MHz}$ (see Fig. 2), which leads to higher line density of the spectrum and complicates its analysis. For completeness, methyl cyanate moreover possess one nucleus with a nonzero electric quadrupole moment $\left({ }^{14} \mathrm{~N}\right)$, however, no quadrupole hyperfine structure has been observed in the rotational spectra.

After eliminating the lines that correspond to the isocyanic acid and methyl isocyanate, the assignment was started with the search for the strongest low- $K_{a}$ transitions using predictions based on the results of Sakaizumi et al. (1995). An example of the predictions of the rotational transitions in the millimeter wave domain is shown in the upper part of Fig. 3. If compared with the experimental spectrum in the middle part of Fig. 3, large discrepancies occur between the observed and predicted frequencies. This is a typical problem of the extrapolation outside the experimentally known data set that makes the confident search in the millimeter wave line surveys impossible. LoomisWood-type plots from AABS package (Kisiel et al. 2005) were very useful for reaching conclusive assignments.

The first set of rotational and centrifugal distortion constants was obtained from the separate fit of the $A$ transitions with standard Watson's $A$-reduced semirigid rotor Hamiltonian (Watson 1977). Next, the $E$ transitions were searched for and added step-by-step, starting at low $J$ and $K_{a}$ and moving up progressively. Both $A$ and $E$ transitions were finally assigned within $J^{\prime \prime}=10-35$ and $K_{a}^{\prime \prime}=0-13$ and analyzed simultaneously using the ERHAM program (Groner 1997, 2012). The structural model from Sakaizumi et al. (1993) was adopted to derive initial values of the internal motion parameters $\rho$ and $\beta$, which represent the magnitude of the $\rho$-vector and the angle between this vector and the principal $a$-axis, respectively. The rotational line sequences of $a$-type $R$-branch transitions for $J^{\prime \prime} \leq 25$ and $K_{a}^{\prime \prime} \leq 8$ were analyzed using the set of the rotational constants, centrifugal distortion constants up to the sixth order, $\rho$ and $\beta$ parameters, first two energy tunneling parameters $\varepsilon_{q}(q=1,2)$, and two tunneling parameters with $q=1$, which were associated with the rotational constants (see Groner 1997, 2012, for definitions and nomenclature of these parameters). Larger discrepancies were found between observed and calculated frequencies for $J^{\prime \prime}>25$ and for even lower $K_{a}^{\prime \prime}$, which could not be rectified with the addition of the tunneling components of the quartic centrifugal distortion constants. These deviations could be partly removed using the tunneling components of the rotational constants with $q=2$. The tunneling parameters associated with the Coriolis operators $J_{a}, J_{a} J^{2}$, or $\left(J_{+}^{2}+J_{-}^{2}\right) J_{a}$ were also applied to decrease those deviations but the $\rho$ and $\beta$ values were significantly changed leading to the unusual value of the moment of inertia of the methyl group. Therefore, these parameters were finally excluded from the fit. The left and middle part of Table 1 present the 25 spectroscopic parameters determined from the nonlinear least-square fit of $390 A$ transitions (290 distinct frequency lines owing to the line blending) and $341 E$ transitions, which lead to the root mean square deviations of $77 \mathrm{kHz}$ and $83 \mathrm{kHz}$ for the sets of $A$ and $E$ transitions, respectively. The moment of inertia of the methyl top, together with other derived internal rotation parameters, are presented on the right side of Table 1 . The experimental frequencies can be found in Table 3, along with the assignments to the rotational quantum numbers $J, K_{a}, K_{c}$ and $v_{\mathrm{obs}}-v_{\text {calc }}$ quantities, where $v_{\text {obs }}$ and $v_{\text {calc }}$ are the observed and calculated frequencies, respectively. The spectroscopic parameters from Table 1 were used to predict the transition frequencies and line strengths multiplied by the square of the corresponding dipole moment component, which are indispensable for a correct molecular identification. These predictions are gathered in Table 4 along with the estimated uncertainties of the predicted frequencies and energies of the lower and upper state energy levels.

\section{Radioastronomical observations}

The new set of the spectroscopic constants of methyl cyanate allow us to attempt a rigorous search for this species in space. The high abundance of molecules containing methoxy $\left(\mathrm{CH}_{3} \mathrm{O}-\right)$ and cyano $(-\mathrm{CN})$ groups in Orion $\mathrm{KL}$ (Tercero et al. 2015; López et al. 2014) and the recent detection of $\mathrm{CH}_{3} \mathrm{NCO}$ as one of the most abundant $\mathrm{CH}_{3}-\mathrm{X}$ species towards the source (Cernicharo et al. 2016) could suggest the presence of methyl cyanate $\left(\mathrm{CH}_{3} \mathrm{OCN}\right)$ in Orion KL. Cernicharo et al. (2016) report an upper limit to its column density of $1 \times 10^{14} \mathrm{~cm}^{-2}$ in Orion KL, according to the IRAM $30 \mathrm{~m}$ data (Tercero et al. 2010). We obtained similar results searching for $\mathrm{CH}_{3} \mathrm{OCN}$ in different positions of the ALMA Science Verification (SV) data of this source. Using the MADEX code (Cernicharo 2012), we derived an upper limit of $2 \times 10^{14} \mathrm{~cm}^{-2}$ in the two emission peaks of $\mathrm{CH}_{3} \mathrm{NCO}$ (see Cernicharo et al. 2016). These positions correspond approximately to the middle of the hot core clumpy structure (see e.g. Favre et al. 2011) 
Hot core
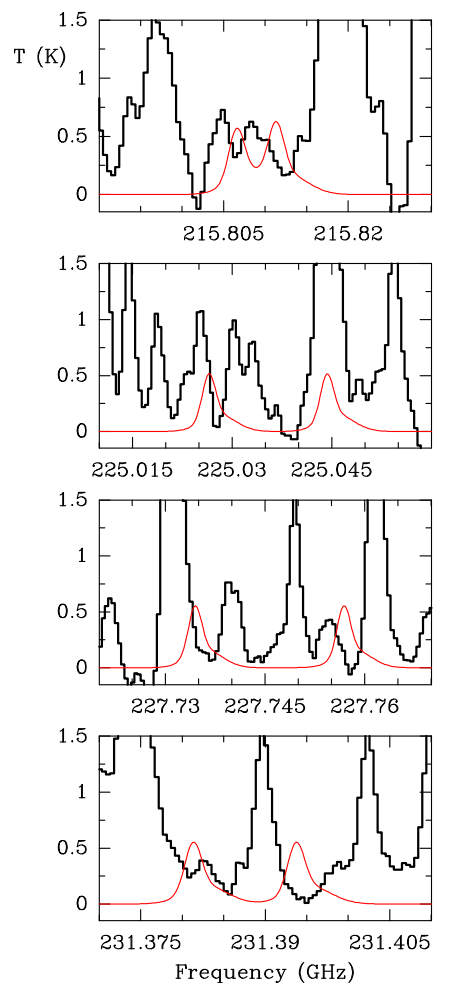

MM4
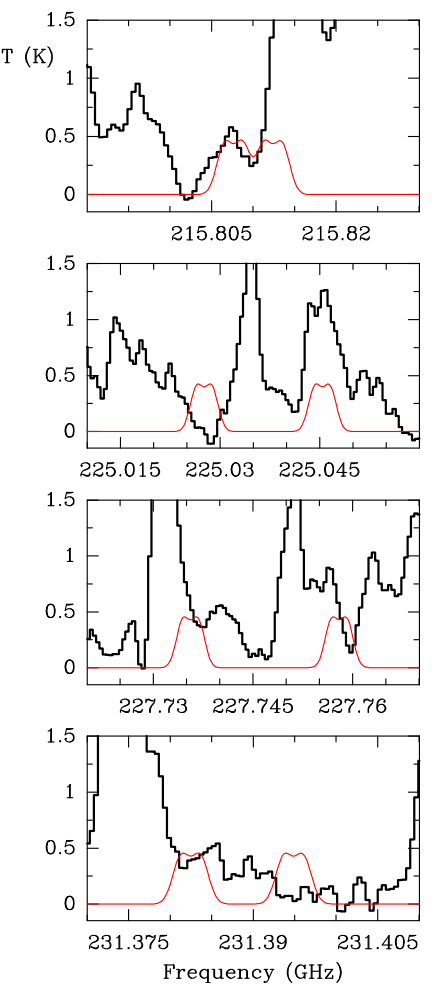

Compact ridge
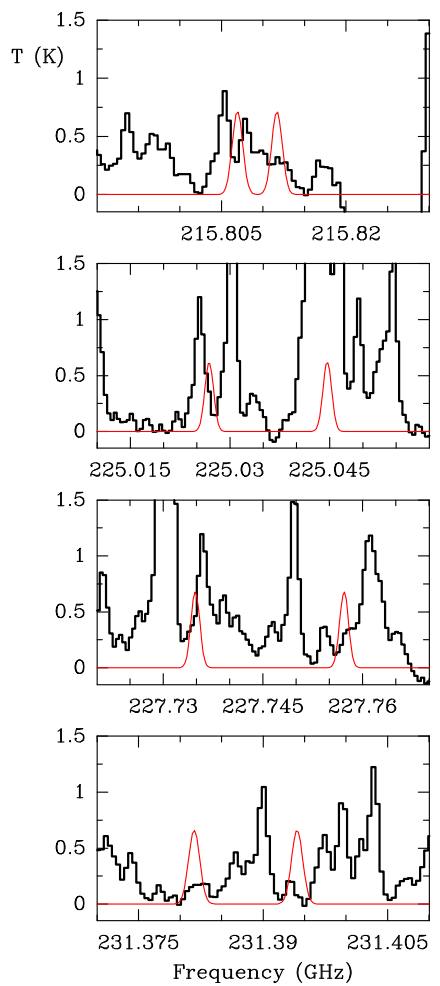

Fig. 4. ALMA SV data of three different positions towards Orion KL at selected frequencies, together with the synthetic spectra obtained using the column densities given in Sect. 4 as upper limits. A $v_{\mathrm{LSR}}$ of $+9.0 \mathrm{~km} \mathrm{~s}^{-1}$ is assumed.

Table 1. Spectroscopic parameters of methyl cyanate in its ground torsional state.

\begin{tabular}{|c|c|c|c|c|c|c|c|c|}
\hline Parameter $^{a}$ & Unit & Value & Parameter $^{b}$ & Unit & Value & Derived parameter ${ }^{c}$ & Unit & Value \\
\hline$A$ & $\mathrm{MHz}$ & $38989.07(20)^{d}$ & $\rho$ & Unitless & $0.186763(67)$ & $I_{\alpha}$ & $\mathrm{u} \AA$ & $3.1962(44)$ \\
\hline$B$ & $\mathrm{MHz}$ & $5322.2520(15)$ & $\beta$ & $\circ$ & $6.822(19)$ & $I_{r}$ & $\mathrm{u} \AA$ & $2.7037(40)$ \\
\hline$C$ & $\mathrm{MHz}$ & $4821.3091(13)$ & $\varepsilon_{1}$ & $\mathrm{MHz}$ & $-286.44(43)$ & $F$ & $\mathrm{~cm}^{-1}$ & $6.2349(92)$ \\
\hline$\Delta_{J}$ & $\mathrm{kHz}$ & $3.40965(66)$ & $\varepsilon_{2}$ & $\mathrm{MHz}$ & $-1.32(12)$ & $\Delta E_{E-A}$ & $\mathrm{MHz}$ & $863.3(15)$ \\
\hline$\Delta_{J K}$ & $\mathrm{kHz}$ & $-81.406(10)$ & {$[A-(B+C) / 2]_{1}$} & $\mathrm{kHz}$ & $1127(29)$ & $\angle(i, a)$ & $\circ$ & $41.232(83)$ \\
\hline$\Delta_{K}$ & $\mathrm{kHz}$ & $1727(23)$ & {$[A-(B+C) / 2]_{2}$} & $\mathrm{kHz}$ & $-126(16)$ & $\angle(i, b)$ & $\circ$ & $48.768(83)$ \\
\hline$\delta_{J}$ & $\mathrm{kHz}$ & $0.79149(89)$ & {$[(B+C) / 2]_{1}$} & $\mathrm{kHz}$ & $-4.76(89)$ & $\angle(i, c)$ & $\circ$ & $90.0^{e}$ \\
\hline$\delta_{K}$ & $\mathrm{kHz}$ & $1.72(11)$ & {$[(B+C) / 2]_{2}$} & $\mathrm{kHz}$ & $-2.08(14)$ & & & \\
\hline$\Phi_{J}$ & $\mathrm{~Hz}$ & $0.01009(28)$ & {$[(B-C) / 4]_{1}$} & $\mathrm{kHz}$ & $4.33(27)$ & & & \\
\hline$\Phi_{J K}$ & $\mathrm{~Hz}$ & $-0.2671(36)$ & {$[(B-C) / 4]_{2}$} & $\mathrm{kHz}$ & $0.62(13)$ & & & \\
\hline$\Phi_{K J}$ & $\mathrm{~Hz}$ & $-8.249(66)$ & $-\left[\Delta_{J}\right]_{1}$ & $\mathrm{~Hz}$ & $1.06(21)$ & & & \\
\hline \multirow[t]{2}{*}{$\phi_{J}$} & $\mathrm{~Hz}$ & 0.00457 (39) & $-\left[\Delta_{J K}\right]_{1}$ & $\mathrm{~Hz}$ & $-35.8(17)$ & & & \\
\hline & & & $-\left[\delta_{K}\right]_{1}$ & $\mathrm{~Hz}$ & $-186.2(95)$ & & & \\
\hline$\sigma_{\mathrm{rms}}$ & $\mathrm{kHz}$ & 95 & & & & & & \\
\hline
\end{tabular}

Notes. ${ }^{(a)}$ Rotational constants $A, B, C$, quartic centrifugal distortion constants $\Delta_{J}, \Delta_{J K}, \Delta_{K}, \delta_{J}, \delta_{K}$, and sextic centrifugal distortion constants $\Phi_{J}$, $\Phi_{J K}, \Phi_{K J}, \phi_{J}$ are given in the notation of Watson's $A$-reduction scheme in $I^{r}$-representation (Watson 1977). ${ }^{(b)}$ The value $\rho$ is the magnitude of the $\rho$-vector, $\beta$ represents the angle between the $\rho$-vector and the principal $a$-axis, $\varepsilon_{1}$ and $\varepsilon_{2}$ are the first and second energy tunneling parameters, respectively. Tunneling parameters associated with the rotational and centrifugal distortion constants are given in the notation $[X]_{q}$, where $X$ is a linear combination of the rotational constants or centrifugal distortion constants and the subscript $q$ designates the tunneling component (see Groner 1997, 2012, for details). ${ }^{(c)} I_{\alpha}$ is the moment of inertia of the methyl top, $I_{r}$ is the reduced moment of inertia, $F$ is the reduced rotational constant, $\Delta E_{E-A}$ is the torsional energy difference, and $\angle(i, a), \angle(i, b), \angle(i, c)$ are the angles between the internal rotation axis $i$ and the principal axis $a, b, c .{ }^{(d)}$ Numbers in parenthesis represent the $1 \sigma$ uncertainty $\left(67 \%\right.$ confidence level) in units of the last decimal digit. ${ }^{(e)}$ Fixed value.

and the MM4 source shown in Wu et al. (2014). According to the $\mathrm{CH}_{3} \mathrm{NCO}$ column densities, we derived the ratio $N\left(\mathrm{CH}_{3} \mathrm{NCO}\right) / N\left(\mathrm{CH}_{3} \mathrm{OCN}\right) \geq 35$ and 20 in the hot core and MM4, respectively. Owing to the richness of the compact ridge in molecules containing the methoxy group (Tercero et al. 2015), we also looked for methyl cyanate in this component, but we did not find this species above the detection limit of the data. Adopting the physical parameters of the compact ridge for trans-ethyl methyl ether derived by Tercero et al. (2015), we found an upper limit to the $\mathrm{CH}_{3} \mathrm{OCN}$ column density of $1 \times 10^{14} \mathrm{~cm}^{-2}$. Figure 4 shows the ALMA SV data of these three positions at selected frequencies, together with the synthetic spectra obtained using the 
Table 2. Physical parameters of the considered cloud cores.

\begin{tabular}{|c|c|c|c|c|c|c|c|}
\hline \multirow[t]{2}{*}{ Source } & \multirow{2}{*}{$\begin{array}{c}v_{\mathrm{LSR}} \\
\left(\mathrm{km} \mathrm{s}^{-1}\right)\end{array}$} & \multirow{2}{*}{$\begin{array}{l}\Delta v_{\mathrm{FWHM}} \\
\left(\mathrm{km} \mathrm{s}^{-1}\right)\end{array}$} & \multirow{2}{*}{$\begin{array}{l}d_{\text {sou }} \\
\left({ }^{\prime \prime}\right)\end{array}$} & \multirow{2}{*}{$\begin{array}{l}T_{\text {rot }} \\
(\mathrm{K})\end{array}$} & \multirow{2}{*}{$\begin{array}{l}N\left(\mathrm{CH}_{3} \mathrm{OCN}\right) \\
\times 10^{14}\left(\mathrm{~cm}^{-2}\right) \\
\end{array}$} & \multicolumn{2}{|c|}{$Q_{\text {rot }}$} \\
\hline & & & & & & A state & E state \\
\hline Orion KL & 8.0 & 3.0 & 3.0 & 150 & $\leq(1.0 \pm 0.3)$ & 8823 & 8825 \\
\hline $\begin{array}{l}\text { (ALMA SV) } \\
\text { Hot core }\end{array}$ & 6.0 & 8.0 & 3.0 & 150 & $\leq(1.0 \pm 0.3)$ & 8823 & 8825 \\
\hline $\begin{array}{l}\text { Orion KL } \\
\text { (ALMA SV) } \\
\text { Compact ridge }\end{array}$ & 7.5 & 2.0 & 3.0 & 100 & $\leq(1.0 \pm 0.3)$ & 5183 & 5185 \\
\hline Orion KL & 8.0 & 3.0 & 3.0 & 150 & $\leq(1.0 \pm 0.3)$ & 8823 & 8825 \\
\hline $\begin{array}{l}\text { (ALMA SV) } \\
\text { MM4 }\end{array}$ & 5.0 & 3.0 & 3.0 & 150 & $\leq(1.0 \pm 0.3)$ & 8823 & 8825 \\
\hline Sgr B2(N) & 63.0 & 7.0 & 2.4 & 200 & $\leq(100 \pm 35)$ & 12300 & 12303 \\
\hline $\begin{array}{l}\text { (IRAM } 30 \mathrm{~m} \text { ) } \\
\text { Hot gas }\end{array}$ & 73.0 & 7.0 & 1.9 & 200 & $\leq(50 \pm 20)$ & 12300 & 12303 \\
\hline Sgr B2(N) & 64.0 & 9.0 & 60.0 & 14 & $<(0.20+0.06)$ & 281 & 281 \\
\hline $\begin{array}{l}\text { (IRAM } 30 \text { m) } \\
\text { Cold gas }\end{array}$ & 75.0 & 12.0 & 60.0 & 14 & $\leq(0.20 \pm 0.06)$ & 281 & 281 \\
\hline $\begin{array}{l}\text { B1-b } \\
\text { (IRAM } 30 \mathrm{~m} \text { ) }\end{array}$ & 6.7 & 0.7 & 60.0 & 12 & $\leq(0.0020 \pm 0.0006)$ & 223 & 223 \\
\hline $\begin{array}{l}\text { TMC-1 } \\
\text { (IRAM } 30 \mathrm{~m} \text { ) }\end{array}$ & 6.0 & 0.7 & 60.0 & 10 & $\leq(0.0020 \pm 0.0006)$ & 170 & 170 \\
\hline
\end{tabular}

column densities given above as upper limits. Above the limit established by these models, it becames clear that lines were missing or mismatched.

As $\mathrm{CH}_{3} \mathrm{NCO}$ has also been detected in $\mathrm{Sgr}$ B2(N) (Halfen et al. 2015; Cernicharo et al. 2016), we modelled the emission of $\mathrm{CH}_{3} \mathrm{OCN}$ according to the physical parameters for $\mathrm{CH}_{3} \mathrm{NCO}$ in Sgr B2(N) (Cernicharo et al. 2016) to search for this species in the public IRAM $30 \mathrm{~m}$ spectrum at $3 \mathrm{~mm}$ of this source (Belloche et al. 2013). Nevertheless, similar to the Orion case, only upper limits to its column density were derived: $1 \times 10^{16} \mathrm{~cm}^{-2}$ and $5 \times 10^{15} \mathrm{~cm}^{-2}$ for the $63 \mathrm{~km} \mathrm{~s}^{-1}$ component and for the second component at $73 \mathrm{~km} \mathrm{~s}^{-1}$, respectively, i.e. a factor $\sim 50$ below that of the column density values of $\mathrm{CH}_{3} \mathrm{NCO}$ (Cernicharo et al. 2016). However, HOCN, an isomer of $\mathrm{HNCO}$, which contains the -OCN group, has been detected in this source (Brünken et al. 2010) so we also expected the presence of methyl cyanate in the cold gas. To explore this possibility, we searched for $\mathrm{CH}_{3} \mathrm{OCN}$ in the previous data, considering the two extended components at $14 \mathrm{~K}$ that were derived by Brünken et al. (2010) for HOCN in Sgr B2(N). Once again, only an upper limit to the column density of $2 \times 10^{13} \mathrm{~cm}^{-2}$ was derived for each component. We also did not find evidence of the presence of this species in the public PRIMOS survey of this source (Neill et al. 2012).

Finally, we concluded this attempt to detect $\mathrm{CH}_{3} \mathrm{OCN}$ in space by searching for this species in dark clouds. We found an upper limit to its column density of $2 \times 10^{11} \mathrm{~cm}^{-2}$ in B1-b and TMC-1 using the data of Cernicharo et al. (2012). Table 2 shows the physical parameters for each cloud adopted for these calculations, as well as the rotational partition function used to derive the upper limits to the $\mathrm{CH}_{3} \mathrm{OCN}$ column density.

Acknowledgements. This paper makes use of the following ALMA data ADS/JAO.ALMA\#2011.0.00009.SV. ALMA is a partnership of ESO (representing its member states), NSF (USA), and NINS (Japan) with NRC (Canada), NSC, and ASIAA (Taiwan), and KASI (Republic of Korea), in cooperation with the Republic of Chile. The Joint ALMA Observatory is operated by ESO, AUI/NRAO, and NAOJ. This work was also based on observations carried out with the IRAM 30-m telescope. IRAM is supported by INSU/CNRS (France), MPG (Germany), and IGN (Spain). The research leading to these results has received funding from the European Research Council under the European Union's Seventh Framework Programme (FP/2007-2013) / ERC-2013-SyG, Grant Agreement n. 610256 NANOCOSMOS, Ministerio de Ciencia e Innovación (Grants CTQ2013-40717-P, AYA2012-32032, and Consolider-Ingenio
2010 CSD2009-00038 program ASTROMOL) and Junta de Castilla y León (Grant VA175U13). J.-C. G. thanks the Centre National d'Études Spatiales (CNES) and the CNRS program Physique et Chimie du Milieu Interstellaire (PCMI) for financial support. C.B. and E.R.A. also wish to thank the Ministerio de Ciencia e Innovacion for an FPI grant (BES-2011-047695 y BES-2014067776).

\section{References}

Belloche, A., Müller, H. S. P., Menten, K. M., Schilke, P., \& Comito, C. 2013, A\&A, 559, A47

Brünken, S., Gottlieb, C. A., McCarthy, M. C., \& Thaddeus, P. 2009, ApJ, 697, 880

Brünken, S., Belloche, A., Martín, S., Verheyen, L., \& Menten, K. M. 2010, A\&A, 516, A109

Cernicharo, J. 2012, in ECLA-2011: Proceedings of the European Conference on Laboratory Astrophysics, Laboratory Astrophysics and Astrochemistry in the Herschel/ALMA Era, 58, 251

Cernicharo, J., Marcelino, N., Roueff, E., et al. 2012, ApJ, 759, L43

Cernicharo, J., Kisiel, Z., Tercero, B., et al. 2016, A\&A, 587, L4

Daly, A., Kolesniková, L., Mata, S., \& Alonso, J. 2014, J. Mol. Spectr., 306, 11 Davies, W., \& Maclaren, J. A. 1951, J. Chem. Soc., 1434

Favre, C., Wootten, H. A., Remijan, A. J., et al. 2011, ApJ, 739, L12

Goesmann, F., Rosenbauer, H., Bredehöft, J. H., et al. 2015, Science, 349, 689

Groner, P. 1997, J. Chem. Phys., 107, 4483

Groner, P. 2012, J. Mol. Spectr., 278, 52

Halfen, D. T., Ilyushin, V. V., \& Ziurys, L. M. 2015, ApJ, 812, L5

Jensen, K. A., Due, M., \& Holm, A. 1965, Acta Chem. Scand., 19, 438

Kisiel, Z., Pszczółkowski, L., Medvedev, I. R., et al. 2005, J. Mol. Spectr., 233, 23

López, A., Tercero, B., Kisiel, Z., et al. 2014, A\&A, 572, A44

Marcelino, N., Cernicharo, J., Tercero, B., \& Roueff, E. 2009, ApJ, 690, L27

Marcelino, N., Brünken, S., Cernicharo, J., et al. 2010, A\&A, 516, A105

Neill, J. L., Muckle, M. T., Zaleski, D. P., et al. 2012, ApJ, 755, 153

Pasinszki, T., \& Westwood, N. P. C. 1995, J. Phys. Chem., 99, 1649

Pasinszki, T., \& Westwood, N. P. C. 2001, J. Phys. Chem. A, 105, 1244

Quan, D., Herbst, E., Osamura, Y., \& Roueff, E. 2010, ApJ, 725, 2101

Sakaizumi, T., Mure, H., Ohashi, O., \& Yamaguchi, I. 1990, J. Mol. Spectr., 140, 62

Sakaizumi, T., Sekishita, K., Furuya, K., et al. 1993, J. Mol. Spectr., 161, 114

Sakaizumi, T., Namikawa, M., \& Ohashi, O. 1995, J. Mol. Struct., 345, 189

Schuurman, M. S., Muir, S. R., Allen, W. D., \& Schaefer, H. F. 2004, J. Chem. Phys., 120, 11586

Tercero, B., Cernicharo, J., Pardo, J. R., \& Goicoechea, J. R. 2010, A\&A, 517, A96

Tercero, B., Cernicharo, J., López, A., et al. 2015, A\&A, 582, L1

Watson, J. K. G. 1977, in Vibrational Spectra and Structure, ed. J. R. Durig (Amsterdam: Elsevier), 6, 1

Wu, Y., Liu, T., \& Qin, S.-L. 2014, ApJ, 791, 123 